\title{
The pattern of eigenfrequencies of radial overtones which is predicted for a specified Earth-model
}

\author{
E. R. LAPWOOD $\left(^{*}\right)-$ R. SATO $(* *)$
}

Received on November 10 th, 1977

INTRODUCTION.

In 1974 Anderssen and Cleary examined the distribution of eigenfrequencies of radial overtones in torsional oscillations of Earth-models. They pointed out that according to Sturm-Liouville theory this distribution should approach asymptotically, for large overtone number $m$, the value $m \pi / \gamma$, where $\gamma$ is the time taken by a shear-wave to travel along a radius from the core-mantle interface to the surface, provided elastic parameters vary continuously along the radius. They found that, for all the models which they considered, the distributions of eigenfrequencies deviated from the asymptote by amounts which depended on the existence and size of internal discontinuities. Lapwood (1975) showed that such deviations were to be expected from Sturm-Liouville theory, and McNabb, Anderssen and Lapwood (1976) extended Sturm-Liouville theory to apply to differential equations with discontinuous coefficients. Anderssen (1977) used their results to show how to predict the pattern of deviations - called by McNabb et al. the solotone effect - for a given discontinuity in an Earth-model.

Recently Sato and Lapwood (1977), in a series of papers which will be referred to here simply as I, II, III, have explored the solotone effect for layered spherical shells, using approximations derived from an exact

(*) Earthquake Research Institute, University of Tokyo, and Emmanuel College, Cambridge.

(**) Geophysical Institute, Faculty of Science, University of Tokyo. 
theory which holds for uniform layering. They have shown how the form of the pattern of eigenfrequencies, which is the graph of $S=\gamma_{m} \omega_{l} / \pi-m$ against $m$, where ${ }_{m} \omega_{l}$ is the frequency of the $m^{t h}$ overtone in the $l^{\text {th }}$ (Legendre) mode of torsional oscillation, is determined as to periodicity (or quasi-periodicity) by the thicknesses and velocities of the layers, and as to amplitude by the amounts of the discontinuities (III). The pattern of eigenfrequencies proves to be extremely sensitive to small changes in layer-thicknesses in a model.

In this paper we examine a proposed Earth-model with six surfaces of discontinuity between core boundary and surface, and predict its pattern of eigenfrequencies. When seismological observations become precise enough, and can be subjected to numerical analysis refined enough, to identify the radial overtones for large $m$, this pattern of eigenfrequencies will prove to be a severe test for any proposed model, including he one which we discuss below.

\section{The Earth-model PEM- $A$.}

We select for our purpose the model PEM-A (parametrically simple Earth-model $A$ ), presented by Dziewonski, Hales and Lapwood (1975), which has the following values at and between discontinuities:

TABLE 1

\begin{tabular}{|c|c|c|c|c|}
\hline & Layer & $\begin{array}{l}\text { Interface } \\
\text { (radius, in } \mathrm{km} \text { ) }\end{array}$ & $\begin{array}{l}\text { Reflection } \\
\text { coefficient }\end{array}$ & $\begin{array}{l}\text { Radial travel-time } \\
\text { within layer (sec.) }\end{array}$ \\
\hline \multirow{6}{*}{ Crust } & 7 & 6.368 .0 & & 3.09859 \\
\hline & 6 & $6,357.0$ & $R_{6}=-0.04491$ & 1.33333 \\
\hline & 5 & $6,352.0$ & $R_{5}=-0.17204$ & 13.10700 \\
\hline & 4 & $6,291.0$ & $R_{4}=0.03484$ & 32.25361 \\
\hline & 3 & $6,151.0$ & $R_{3}=-0.01360$ & 42.77049 \\
\hline & 2 & 5.951 .0 & $R_{2}=-0.05425$ & 47.17423 \\
\hline \multirow{2}{*}{$\begin{array}{l}\text { Lower } \\
\text { mantle }\end{array}$} & 1 & 5.701 .0 & $R_{i}=-0.09085$ & 327.80071 \\
\hline & & $3,485.7$ & & \\
\hline
\end{tabular}

(Note that the numbering of layears and reflection coefficients proceeds upwards from the core boundary). 
The reflection coefficient $R$, is defined as

$$
R_{j}=\frac{\rho_{i+1}^{\prime} \beta_{j+1}^{\prime}-\rho_{i} \beta_{i}}{\rho_{i+1}^{\prime} \beta_{j+1}^{\prime}+\rho_{i} \beta_{i}}
$$

where $\rho_{i}$ is density and $\beta_{i}$ is shear velocity adjacent to the $(j, j+1)$ interface in the $j^{\text {th }}$ layer and $\rho^{\prime}{ }_{i+1}, \beta^{\prime}{ }_{j+1}$ are adjacent to the same interface in the $(j+1)^{i t h}$ layer. Since density and shear velocity increase downwards in PEM-A, except at the $(4,5)$ boundary, all reflection coefficients but one are negative.

The patterin of Eigenfrequencies.

Lapwood and Sato proved (III, § 2) that for a shell of three uniform layers, the $j^{\text {th }}$ being bounded by spherical surfaces $r=r_{1-1}$ and $r=r_{j}$, a measure of the solotone effect is given for any $\omega$ by

$$
\begin{gathered}
S=\gamma \omega / \pi-m \\
\doteqdot \frac{R_{1}}{\pi} \sin 2 \chi_{1}-\frac{R_{2}}{\pi} \sin 2 \chi_{3},
\end{gathered}
$$

where $R$, is the reflection coefficient at normal incidence of a ray from region (i) on the $(j, j+1)$ interface, and $\chi_{1}$ is the change of phase as a shear-wave passes through the $j^{t h}$ layer. $\chi_{i}=\omega \chi_{i}^{\prime}$, where

$$
\chi_{i}^{\prime}=\left(T-\frac{d T}{d \Delta} \Delta\right) j^{t h} \text { layer, }
$$

$\Delta$ and $T$ for any arc being the angle subtended at the centre of the sphere and the time taken on the arc respectively.

As shown in the Appendix, the extension of the formula [3] to a shell of $n$ layers is

$$
S \doteqdot-\frac{1}{\pi} \sum_{j=1}^{n-1} R_{j} \sin \left(\frac{2 m \pi}{\gamma} \sum_{i=j+1}^{n} \chi_{p}^{\prime}\right),
$$

where

$$
\gamma=\sum_{j=1}^{\prime \prime} \chi_{i}^{\prime}
$$


We have computed $S$ from [5] for $l=2$ and $m=1,2, \ldots, 150$. The values of $S$ are graphed against $m$ in Fig. 1. Our earlier work has shown that $S$ is rather insensitive to the value of $l(I I I, \S \S 1,2)$, so that the pattern of eigenfrequencies which emerges from Fig. 1 may be said to characterise the radial overtones of all modes of torsional oscillations of the model.

We observe immediately that in Fig. 1 there is a pattern, of period 10 in $m$, which changes slowly as $m$ proceeds from 1 to 150 . We have taken this large range of $m$ to exhibit another period of $S$, which is shown in the long sinusoidal arc of period about 110 . We now look for the explanation of these two recurrence periods.

\section{ANALYSiS OF SOLOTONE FUNCTION $S$.}

If into the general formula [5] we insert the values defined for the Earth-model PEM-A by the table 1, and retain only such precision as is adequate to our propose, we get

$$
\begin{aligned}
S & =\frac{1}{\pi}\{0.091 \sin 2 m \pi(0.2989)+0.054 \sin 2 m \pi(0.1980) \\
& +0.014 \sin 2 m \pi(0.1065)-0.035 \sin 2 m \pi(0.0375) \\
& +0.172 \sin 2 m \pi(0.0095)+0.045 \sin 2 m \pi(0.0066)\} .
\end{aligned}
$$

The coefficients of the sines are $R_{1}, R_{2}, \ldots, R_{0}$ respectively. The largest amplitudes are those of the fifth and first terms, while the contribution of the third term is very small.

If we now ask what lowest values of $m$ will produce in the successive arguments of the sines almost exact multiples of $2 \pi$, we find respectively

$$
10,10,10,80,105,150 .
$$

Thus the first three terms of [7] have the same sign and almost the same period in $m$, and together account for the dominant period of 10 in $m$ in the pattern of eigenfrequencies shown in Fig. 1. The first term contains $R_{1}$, which is the coefficient of reflection at the $667 \mathrm{~km}$ discontinuity; the second contains $R_{2}$, which is the coefficient of reflection 
at the $417 \mathrm{~km}$ discontinuity, while the third contains $R_{3}$, corresponding to a small discontinuity at $217 \mathrm{~km}$.

The fifth term has $R_{5}$ as factor, corresponding to reflection at the base of the crust. Thus the shape of the pattern of eigenfrequencies seems to be determined mainly - in fact almost completely - by resonances among reflections at the $667 \mathrm{~km}$ and $417 \mathrm{~km}$ discontinuities and the Moho.

We verify these statements by computing, firstly,

$$
\begin{aligned}
S^{*}=\frac{1}{\pi}\{0.0909 \sin m( & 2 \pi \times 0.2989) \\
& +0.0543 \sin m(2 \pi \times 0.1980)\}
\end{aligned}
$$

for $m=1,2, \ldots, 20$, and secondly

$$
S^{\prime}=\frac{1}{\pi}\{0.17204 \sin m(2 \pi \times 0.00947928)\}
$$

for $m=5,10,15, \ldots, 120$.

Fig. 2 shows the graph of $S^{*}$ against $m$. We see that the essential shape of the small pattern of period 10 in Fig. 1 is reproduced in Fig. 2, though Fig. 2 inevitably shows no evidence of the longer arc on which, the small patterns lie in Fig. 1.

Fig. 3 shows the values of $S^{\prime}$ lying on a sine curve of period 105 in $m$. In the same figure are plotted averages of eigenvalues in successive sets of 10. The locus of averages eliminates the small patterns. There is very good agreement between the two graphs. One would not expect perfect agreement, since the period 10 is not exact for any of the first three terms of [7]. The lengthening of the recurrence period in the graph of averages to about 112 is probably mainly due to the last term of [7], which arises from reflection within the crust.

We may compare Figs. 1 and 2 with Figs. $3 \mathrm{a}$ and $3 \mathrm{~b}$ of III, which show the pattern of eigenfrequencies for an averaged PEM-A, which has discontinuities only at depths $417 \mathrm{~km}$ and $667 \mathrm{~km}$. Although the velocity distributions and consequently the coefficients of reflection differ for the two models, the dominant recurrence period of 10 in $\mathrm{m}$ shows up clearly in both. 

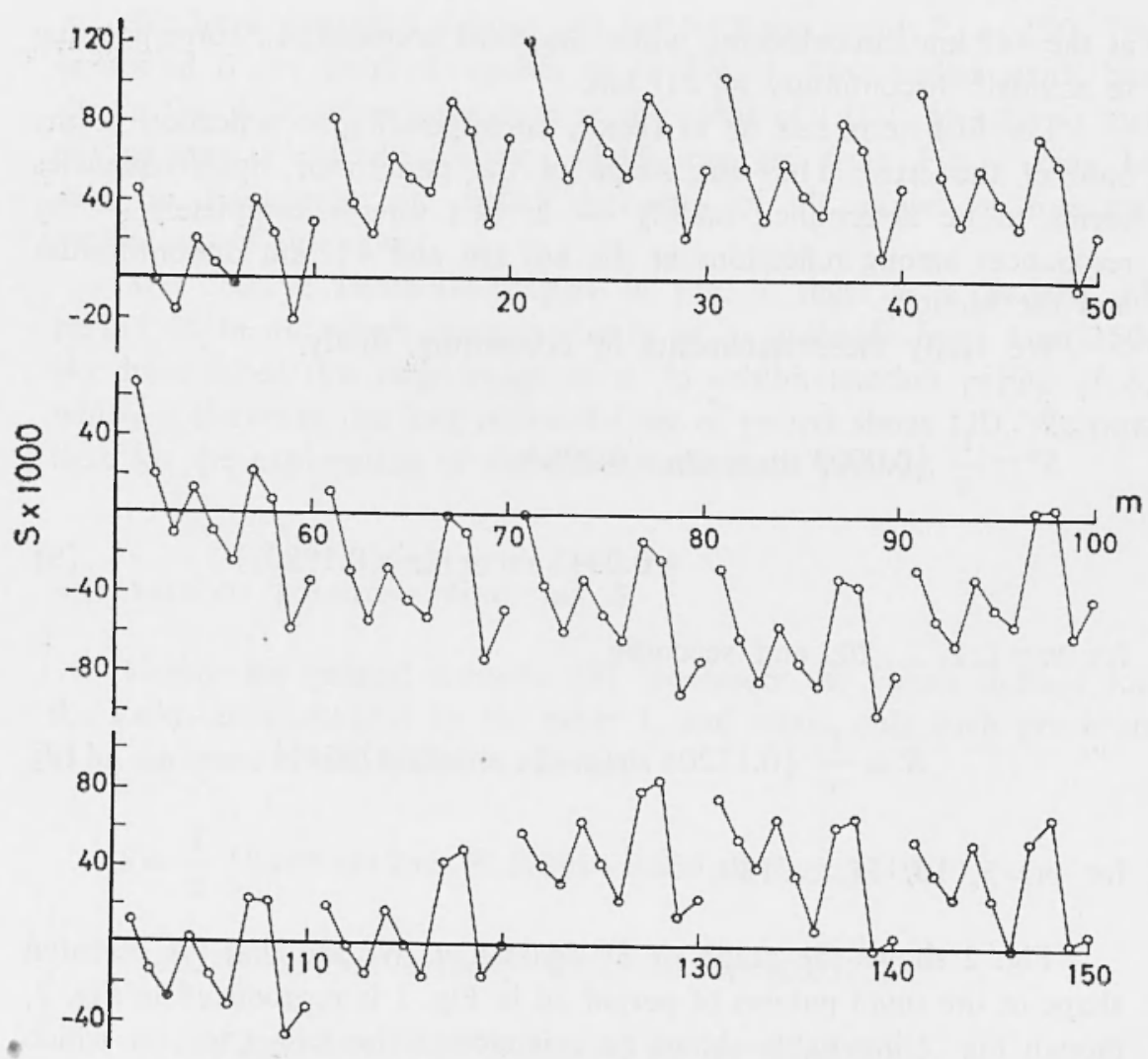

Fig. 1. Graph of $S=\gamma \omega / \pi-m$ against $m$ for $m=1,2,3, \ldots, 150$.

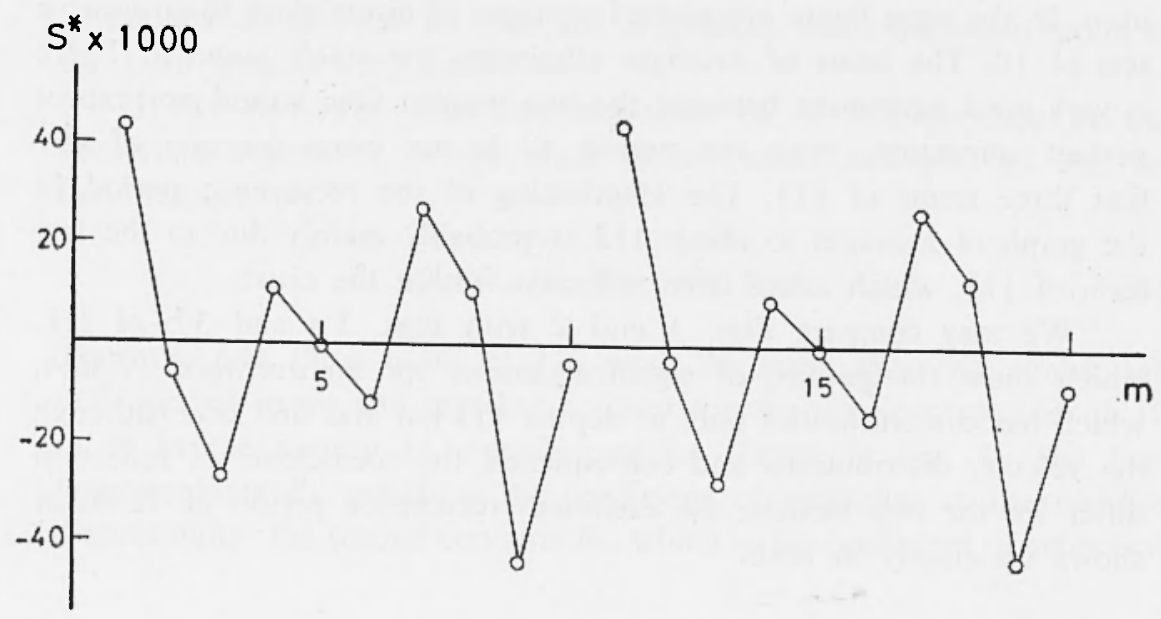

Fig. 2. Graph of $S^{*}$ (given by [9] against $m$ for $m=1,2, \ldots, 20$. 


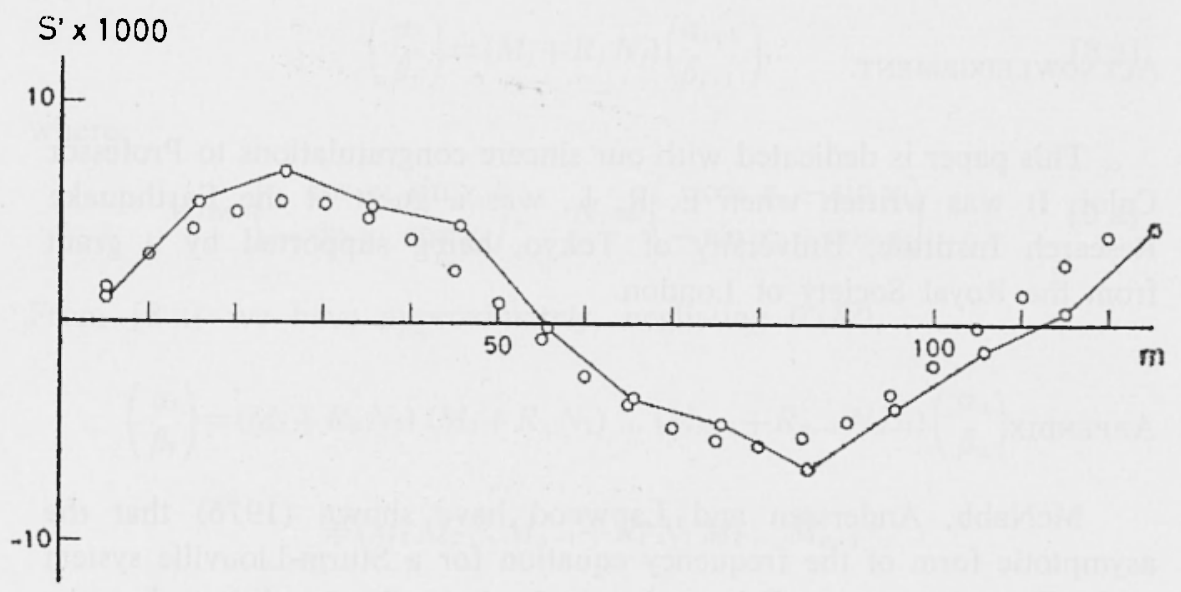

Fig. 3. Graph of $S^{\prime}$ (given by [10] against $m$ for $m=5,10,15, \ldots, 120$ (sine curve), compared with graph of averages over groups of 10 (broken line).

In the formulae [5] and [7] multip'e internal reflections have been neglected. In [9] and [10] radial travel times have been used instead of $\chi_{p}{ }^{\prime}$ of [5]. For these reasons our exposition must be seen as only a first order approximation. Nevertheless, since the second order effects are all small we may be confident that even when superposed they will not affect our main conclusions: in our previous papers we compared $m \pi / \gamma+S$ with $m \omega_{l}$ computed with great precision from exact frequency equations, and found excellent agreement.

\section{Conclusions.}

We have found the pattern of eigenfrequencies for the Earth-model PEM-A, and have shown that it is determined mainly by the depth of the Moho and the depths of discontinuities in the neighbourhood of $400 \mathrm{~km}$ and $650 \mathrm{~km}$. When it becomes possible, with increased accuracy of observation and increased delicacy of computational analysis, to identify and measure the higher overtones of torsional oscillations of the Earth itself, the predicted pattern of eigenfrequency can be put to the test. Then the model will receive additional strong support, or else will be shown to need modification. 
ACKNOW LEDGEMENT.

This paper is dedicated with our sincere congratulations to Professor Caloi. It was written when E. R. L. was a guest at the Earthquake Research Institute, University of Tokyo, being supported by a grant from the Royal Society of London.

ApPEND1X.

McNabb, Anderssen and Lapwood have shown (1976) that the asymptotic form of the frequency equation for a Sturm-Liouville system with discontinuous coefficients is obtained as the condition that the following set of homogeneous linear equations is consistent:

$$
\begin{gathered}
\alpha_{1}=0, \\
\left.\begin{array}{cc}
\alpha \sin \chi_{i}+\beta_{i} \cos \chi_{i} & -\gamma_{i} \hat{\mu}_{j+1}=0, j=1,2, \ldots, n-1 \\
\alpha_{i} \cos \chi_{i}-\beta_{i} \sin \chi_{i}-\frac{1}{\gamma_{i}} \alpha_{j+1}=0,
\end{array}\right\} \\
\alpha_{n} \cos \chi_{n}-\beta_{n} \sin \chi_{n}=0,
\end{gathered}
$$

where in our problem $\chi_{i}$ is defined as in $\S 3$ and

$$
\gamma_{i}^{2}=\beta_{i} \rho_{i} / \beta_{i+1} \rho_{i+1}
$$

From [1],

$$
R_{j}=\left(1-\gamma_{j}^{2}\right) /\left(1+\gamma_{i}^{2}\right)
$$

so that if we neglect terms of order $R_{j}{ }^{2}$

$$
\gamma_{j} \doteqdot 1-R_{i}, \quad \frac{1}{\gamma_{i}} \doteqdot 1+R_{j}
$$

Inserting these into [2 a] and inverting the equations we get, to first order in $R_{i}$, 


$$
\left(\begin{array}{c}
\alpha_{j} \\
\beta_{j}
\end{array}\right)=\left(M_{j}+R_{i} N_{j}\right)\left(\begin{array}{c}
\alpha_{j+1} \\
\beta_{i+1}
\end{array}\right),
$$

where

$$
M_{j} \equiv\left\{\begin{array}{r}
\cos \chi_{i} \sin \chi_{i} \backslash \\
-\sin \chi_{i} \cos \chi_{i}
\end{array}\right), \quad N_{i} \equiv\left(\begin{array}{r}
\cos \chi_{i}-\sin \chi_{i} \\
-\sin \chi_{j}-\cos \chi_{j}
\end{array}\right)
$$

From [8 a] we have approximately, neglicting $0\left(R^{2}\right)$,

$$
\begin{gathered}
\left(\begin{array}{c}
\alpha_{1} \\
\beta_{1}
\end{array}\right)=\left(M_{1}+R_{1} N_{1}\right)\left(M_{1}+R_{2} N_{2}\right) \ldots\left(M_{n-1}+R_{n-1} N_{n-1}\right)\left(\begin{array}{c}
\alpha_{n} \\
\beta_{n}
\end{array}\right) \\
\doteqdot\left(M_{1} M_{2} \ldots M_{n-1}+R_{1} N_{1} M_{2} \ldots M_{n-1}\right. \\
\left.+M_{1} R_{2} N_{2} M_{3} \ldots M_{n-1}+\ldots+M_{1} M_{2} \ldots R_{n-1} N_{n-1}\right)\left(\begin{array}{c}
\alpha_{n} \\
\beta_{2}
\end{array}\right) .
\end{gathered}
$$

If for $M_{j}$ we write $M\left(\chi_{j}\right)$ and for $N_{j}$ write $N\left(\chi_{j}\right)$, then we easily prove by matrix multiplication that

$$
\begin{aligned}
& M_{1} M_{2}=M\left(\chi_{1}+\chi_{2}\right), \\
& M_{1} N_{2}=N\left(\chi_{1}+\chi_{2}\right),
\end{aligned}
$$

and

$$
N_{1} M_{2}=N\left(\chi_{1}-\chi_{2}\right)
$$

Thus

$$
\begin{gathered}
M_{1} M_{2} \ldots M_{n-1}=M\left(\chi_{1}+\chi_{2}+\ldots+\chi_{n-1}\right) \\
M_{1} N_{2} \ldots M_{n-1}=N\left(\chi_{1}+\chi_{2}-\chi_{3} \ldots-\chi_{n-1}\right), \\
M_{1} M_{2} \ldots N_{n-1}=N\left(\chi_{1}+\chi_{2}+\ldots+\chi_{n-1}\right) .
\end{gathered}
$$

Re-writing $[10 \mathrm{a}]$ as

$$
\left(\begin{array}{l}
\alpha_{1} \\
\beta_{1}
\end{array}\right)=\left(\begin{array}{ll}
P^{11} & P^{12} \\
P^{21} & P^{22}
\end{array}\right)\left(\begin{array}{l}
\alpha_{n} \\
\beta_{n}
\end{array}\right)
$$

we get, from $\alpha_{1}=0$,

$$
P^{11} \alpha_{n}+P^{12} \beta_{n}=0 \text {, }
$$


and from [4 a]

$$
P^{11} \sin \chi_{n}+P^{12} \cos \chi_{n}=0 \text {. }
$$

But $P^{11}=\left\{M\left(\sum_{1}^{n-1} \chi_{j}\right)\right\}^{11}+\left\{\sum_{j=1}^{n-1} R_{j} N\left(\chi_{1}+\ldots+\chi_{j}-\chi_{j+1}-\ldots-\chi_{n-1}\right)\right\}^{11}, \quad[20 \mathrm{a}]$ and similarly for $P^{12}$.

Hence from [19a]

$$
\begin{aligned}
& \sin \chi_{n}\left\{\cos \phi+\sum_{j=1}^{n-1} R_{1} \cos \psi_{l}\right\}+ \\
& +\cos \chi_{n}\left\{\sin \phi+\sum_{j=1}^{n-1} R_{j}\left(-\sin \psi_{j}\right)\right\}=0,
\end{aligned}
$$

where

$$
\begin{gathered}
\phi=\chi_{1}+\chi_{2}+\ldots+\chi_{n-1}, \\
\psi_{i}=\chi_{1}+\chi_{2}+\ldots+\chi_{i}-\chi_{i+1}-\ldots-\chi_{n-1}
\end{gathered}
$$

i. e.

$$
\sin \left(\chi_{1}+\chi_{2}+\ldots+\chi_{n}\right)-\sum_{j=1}^{n-1} R_{j} \sin \left(\psi_{j}-\chi_{n}\right)=0 .
$$

If we neglect $R_{j}$, our zero order approximation is

$$
\chi_{1}+\chi_{2}+\ldots+\chi_{n}=m \pi \text {, where } m \text { is any integer. }
$$

Let the first order approximation be

$$
\chi_{1}+\chi_{2}+\ldots+\chi_{n}=m \pi+\delta,
$$

so that

$$
\hat{o}=\omega \gamma-m \pi \text { and } S=\delta / \pi \text {. }
$$

Substituting [26a] into [24a] we get

$$
(-1)^{m} \sin \delta=\sum_{j=1}^{n-1} R_{j} \sin \left(m \pi+\delta-2 \sum_{p=j+1}^{n} \chi_{p}\right)
$$


If we regard $\delta$ as small and neglect it in the expressions on the righthand side we obtain

$$
S \doteqdot-\frac{1}{\pi} \sum_{j=1}^{n-1} R_{j} \sin 2 \sum_{p=j+1}^{n} \chi_{p}
$$

Now writing $\chi_{p}=\omega \chi_{p}{ }^{\prime}$, and $\omega=m \pi / \gamma$ in the small terms, we get

$$
S=-\frac{1}{\pi} \sum_{j=1}^{n-1} R_{i} \sin \frac{2 m \pi}{\gamma} \sum_{p=j+1}^{n} \chi_{p}^{\prime} .
$$

\section{REFERENCES}

ANDersSen, R. S., 1977. Geophys. J. R. astr. Soc., 50, 303.

Andersssen, R. S., and Cleary, J. R., 1974. Geophys. J. R. astr. Soc., 39, 241.

Dziewonski, A. M., Hales, A. L., and Lapwood, E. R., 1975. Phys. Earth and Plan. Inst., 10, 12.

LAPWOOD, E. R., 1975. Geophys. J. R. astr. Soc., 40, 453.

Lapwood, E. R. and Sato, R., 1977. (III). J. Phys. Earth 25, 361.

Lapwood, E. R. and Sato, R., 1977. J. Computational Physics 29, 412.

Mc Nabb, A., Anderssen, R. S., and Lapwood, E. R., 1976. J. Math. Anal. Appl. 741.

Sato, R. and Lapwood, E. R., 1977. (I). J. Phys. Earth 25, 257.

Sato, R. and LaPwood, E. R., 1977. (II). J. Phys. Earth 25, 345. 